\title{
MOTIVACIÓN POR ESTUDIAR CARRERAS DE INGENIERÍA: UN ESTUDIO CON PERSPECTIVA DE GÉNERO EN LA FACULTAD DE INGENIERÍA DE LA UNIVERSIDAD NACIONAL DE LA PLATA
}

MOTIVAÇÃO PARA ESTUDAR CARREIRAS DE ENGENHARIA: UM ESTUDO COM PERSPECTIVA DE GÊNERO NA FACULDADE DE ENGENHARIA DA UNIVERSIDADE NACIONAL DE LA PLATA

MOTIVATION TO STUDY ENGINEERING CAREERS: A STUDY WITH A GENDER PERSPECTIVE AT THE FACULTY OF ENGINEERING AT THE NATIONAL UNIVERSITY OF LA PLATA

\author{
Viviana Angélica Costa \\ Universidad Nacional de La Plata, Argentina \\ María Valeria Calandra \\ Universidad Nacional de La Plata, Argentina \\ Juana Inés Gallego Sagastume \\ Universidad Nacional de La Plata, Argentina
}

\section{RESUMEN}

Este trabajo se centra en la baja proporción de jóvenes, que estudian carreras de ciencias e ingeniería. En particular, el foco está puesto dentro de ese grupo en la tasa de mujeres que ingresan a las carreras de ciencias e ingeniería (STEM). Esto es el efecto, creemos, de una problemática mayor que genera la brecha de género y la baja accesibilidad de las mujeres a la educación y en especial a la educación en ciencias. Desde un grupo de investigación en educación en ciencias de la Facultad de Ingenieria de la Universidad Nacional de La Plata se pretende hacer un aporte al estudio de este fenómeno. Se construye un cuestionario como instrumento para obtener datos acerca de la motivación que manifiestan un grupo de estudiantes de esa facultad por la elección de sus carreras. Los resultados indicarían que la escuela secundaria y la familia son los ámbitos educativos y sociales que más influirían en la elección de los jóvenes en general, y de las mujeres en particular, por estudiar carreras de ingeniería.

\begin{tabular}{|l|l|l|l|l|l|} 
Revista RBBA & ISSN 2316-1205 & Vitória da Conquista & V.9 $\mathrm{n}^{\mathrm{o}} 1$ & p. 15-33 & Julho/2020 \\
\hline
\end{tabular}




\title{
MOTIVACIÓN POR ESTUDIAR CARRERAS DE INGENIERÍA: UM ESTUDIO CON PERSPECTIVA DE GÉNERO EN LA FACULTAD DE INGENIERÍA DE LA UNIVERSIDAD NACIONAL DE LA PLATA
}

Además se encuentra que no tienen modelos femeninos a seguir que les sean de referentes para estas carreras. Lo observado serviría de base para generar estrategias educativas, políticas y sociales que colaboren con una mayor accesibilidad, motivación e interés por parte de las mujeres en estudiar carreras de ciencias e ingeniería.

Palabras clave: Ingeniería. Mujeres. Género. América Latina.

\section{RESUMO}

Este trabalho concentra-se na baixa proporção de jovens que estudam cursos em ciências e engenharia. Em particular, o foco é colocado nesse grupo na taxa de mulheres ingressando em carreiras em ciências e engenharia (STEM). Esse é o efeito, acreditamos, de um problema maior que gera a brecha de gênero e a baixa acessibilidade das mulheres à educação e, principalmente, à ciência. De um grupo de pesquisa em ensino de ciências da Faculdade de Engenharia da Universidade Nacional de La Plata, pretende-se contribuir para a análise desse fenômeno. Um questionário é construído como um instrumento para obter dados sobre a motivação expressa por um grupo de estudantes da Faculdade para a escolha de suas carreiras. Os resultados indicariam que o ensino médio e a família são as esferas educacionais e sociais que mais influenciam a escolha dos jovens em geral, e de mulheres em particular, para estudar carreiras de engenharia. Além disso, verifica-se que elas não têm modelos femininos para se referir a essas carreiras. $\mathrm{O}$ que foi observado serviria de base para gerar estratégias educacionais, políticas e sociais que colaborem com maior acessibilidade, motivação e interesse das mulheres em estudar carreiras em ciências e engenharia.

Palavras-chave: Engenharia. Mulheres. Gênero. América Latina.

\begin{abstract}
This work focuses on the problem at a global level about the low number of young people, in particular women, who study science and engineering careers. This is part of a larger problem that has to do with the gender gap and the low accessibility of women to education and, especially, science education. Based on a research group on science education at the Faculty of Engineering of the National University of La Plata, we intend to contribute to discuss this phenomenon, for which a questionnaire was built as an instrument to obtain data about the motivation expressed by a group of students from the mentioned Faculty to choose their careers. The results indicate that high school and the family are the educational and social areas that most influence the choice of young women in general, and women in particular, to study engineering careers. In addition, it appears that they do not have female models of reference for their choices. What was observed will serve as a basis to generate educational, political and / or social strategies that collaborate with greater accessibility, motivation and interest on the part of women in the study of science and engineering careers.
\end{abstract}

Keywords: Engineering. Women. Genre. Latin America. 


\section{Introducción}

La UNESCO (2019) en sus informes más recientes menciona que el acceso a la educación para niñas y jóvenes en las áreas de ciencia, tecnología, ingeniería y matemática (STEM, por sus siglas en inglés) ha mejorado a nivel mundial, pero que aún persisten importantes inequidades en los diversos niveles, locales, de país y regionales (GARCÍA, GONZÁLEZ, OVIEDO, 2017). STEM es un enfoque pedagógico de enseñanza transdisciplinar, surgido cerca del año 2009 en Estados Unidos, basado en proyectos que integra la ciencia, la tecnología, la ingeniería y la matemática con el objetivo de lograr un aprendizaje contextualizado y significativo, enseñando con problemas reales, incorporando tecnologías y articulando contenidos a estudiar con otras ciencias e ingeniería, entre otros.

El informe menciona que las diferencias de género en estas disciplinas se presentan en todos los niveles de educación. Al llegar a la educación superior, las mujeres representan solo el 35\% de los estudiantes matriculados en el estudio de áreas relacionadas con STEM. También son visibles las brechas de género en las ciencias que muchas de las veces se entienden principalmente como desigualdades. Las mujeres aún son minoría en las áreas de la física y las ingenierías, sobre todo en las relacionadas con ciencias de la computación e informática. En relación a la trayectoria profesional de las mujeres, en América Latina y el Caribe, un promedio de $45 \%$ se dedican a la investigación. Si bien este porcentaje promedio muestra una relativa paridad de género en la investigación científica profesional, aún es necesario mirar con mayor detenimiento las condiciones en las que trabajan, y las dificultades que tienen las mujeres para acceder a puestos superiores.

En Arias (2017) se señala que los porcentajes de universitarias matriculadas en ciencias naturales, matemáticas y estadísticas oscilan desde 53 a $59 \%$ en Brasil, a menos del 47\%, en Argentina y Chile. En México y Colombia, este rango va del 47 al 53\%. La matrícula de alumnas inscritas en programas de ingeniería, manufactura y construcción en educación superior en Brasil supera el 35\%; en Honduras y Colombia, es entre 29 y 35\%, y en México, entre 25 y $29 \%$.

De acuerdo con Arango Gaviria (2006) la ingeniería es una de las profesiones más valoradas, pero tiene los porcentajes más bajos de mujeres y proyecta una imagen fuertemente masculina. Esta autora comenta en su artículo que la socióloga australiana Judy Wajcman opina 


\section{MOTIVACIÓN POR ESTUDIAR CARRERAS DE INGENIERÍA: UM ESTUDIO CON PERSPECTIVA DE GÉNERO EN LA FACULTAD DE INGENIERÍA DE LA UNIVERSIDAD NACIONAL DE LA PLATA}

que, la ingeniería, es un ejemplo interesante de cultura masculina, pues se encuentra en los límites entre el trabajo físico e intelectual.

Gloria Bonder (2002), coordinadora de la Cátedra UNESCO, Mujer, Ciencia y Tecnología en América Latina, menciona que hay diversos factores que pueden explicar estas disparidades que, si bien han ido disminuyendo en la región, aún son sumamente particulares según el país. Además, menciona que "la ciencia, la tecnología y la innovación son espacios de poder", así como la política, la academia y la investigación han sido espacios de poder masculino y prestigio. Cambiar esos patrones es afectar los intereses de quienes han ocupado históricamente esos espacios. En sus palabras, los estereotipos en el imaginario social también influyen, pues la imagen del científico se suele representar en figura de varones, con características particulares, y recién ahora se empiezan a visibilizar perfiles de mujeres científicas que, además, rompen con esos estereotipos.

Según la UNESCO, los estereotipos de género retratados en los medios son internalizados por los niños, las niñas y los adultos, y afectan la forma en que ellos se ven a sí mismos y a los demás.

Medidas orientadas a promover la igualdad de género, tales como leyes de transversalidad de género o políticas como cuotas, incentivos financieros u otros, pueden aumentar la participación de niñas y mujeres en la educación y las carreras STEM, señala la UNESCO.

Bonder, también menciona que "En este momento la región vive una ola conservadora, que puede provocar un retroceso en los derechos para las mujeres, así como para el avance de la ciencia. Hay muchos países que han trabajado por la igualdad de género, como Argentina, Uruguay, Chile, Brasil, México, Colombia, Costa Rica; es importante seguir apoyando los movimientos no solo por las científicas y las investigadoras, sino por un tema que ha afectado la agenda del discurso público, y que se resume en igualdad".

En esta línea, además en el año 2015 los 193 Estados Miembros de la ONU aprobaron la Agenda para el Desarrollo Sostenible 2030, que establece 17 Objetivos de Desarrollo Sostenible (ODS). Se basa en un plan de acción en favor de las personas, el planeta y la prosperidad, promoviendo a su vez la paz y la libertad. Este plan consta de 17 metas, entre las que en relación a la problemática aquí mencionada destacamos las siguientes:

\begin{tabular}{l|l} 
Revista RBBA & Revista Binacional Brasil Argentina
\end{tabular} 


\section{MOTIVACIÓN POR ESTUDIAR CARRERAS DE INGENIERÍA: UM ESTUDIO CON PERSPECTIVA DE GÉNERO EN LA FACULTAD DE INGENIERÍA DE LA UNIVERSIDAD NACIONAL DE LA PLATA}

$\checkmark$ Garantizar una educación inclusiva, equitativa y de calidad y promover oportunidades de aprendizaje durante toda la vida para todos.

$\checkmark$ Eliminar las disparidades de género en la educación y asegurar el acceso igualitario a todos los niveles de la enseñanza y la formación profesional para las personas vulnerables, incluidas las personas con discapacidad, los pueblos indígenas y los niños en situaciones de vulnerabilidad.

Lograr la igualdad entre los géneros y empoderar a todas las mujeres y las niñas.

$\checkmark$ Asegurar la participación plena y efectiva de las mujeres y la igualdad de oportunidades de liderazgo a todos los niveles decisorios en la vida política, económica y pública.

En América Latina, García-Holgado, Camacho Díaz, y García-Peñalvo (2019) mencionan en su trabajo la brecha de género existente en el sector STEM en América Latina y presentan una propuesta europea que tiene como objetivo mejorar las estrategias y los mecanismos de atracción, acceso y orientación de las mujeres en los programas de educación superior STEM en América Latina.

En México, Ruiz, Córdoba, Salas y Wienner (2016) presentan un trabajo sobre la motivación de las mujeres por las carreras de ingeniería y tecnología, e indagan sobre el modelo de desempeño futuro de las estudiantes mujeres inscritas en carreras de ingeniería, en el municipio de Mexicali. Muestran que no existe un modelo femenino a seguir, por lo que proponen la inclusión de un modelo STEM, en su institución educativa para incrementar la inclusión, preferencia y matrícula de mujeres en esta área del conocimiento.

En Argentina, el Consejo Federal de Decanos de las Facultades de Ingeniería (CONFEDI) como aporte para atacar la problemática mencionada, durante el año 2019 ha editado un libro al que denominó "Efecto Matilda”. ¿Por qué Matilda? Porque se refiere a la insuficiente valoración que sistemáticamente se hace de las mujeres en el campo científico y al escaso reconocimiento a sus contribuciones académicas (ROSSITER, 1993). Esto también se relaciona al fenómeno por el cual las mujeres y sus aportes científicos se acreditan a los hombres o, directamente, se pasan por alto por completo. En el libro se relatan varias historias de mujeres que han sido referentes en los campos de la ciencia e ingeniería en Argentina. 


\section{MOTIVACIÓN POR ESTUDIAR CARRERAS DE INGENIERÍA: UM ESTUDIO CON PERSPECTIVA DE GÉNERO EN LA FACULTAD DE INGENIERÍA DE LA UNIVERSIDAD NACIONAL DE LA PLATA}

Una referente en Latinoamérica, pionera de la inserción de la mujer en el ámbito de la Ingeniería, fue la argentina Elisa Beatriz Bachofen, nacida en la ciudad de Buenos Aires. Fue la primera mujer diplomada en ingeniería civil en América del Sur, habiendo estudiado en la Facultad de Ciencias Exactas, Físicas y Naturales de la Universidad de Buenos Aires, donde se graduó en 1917 con una tesis que aportaba desde un punto de vista productivo a la Instalación de una fábrica de hilados y tejidos utilizando algodón del Chaco. Además fue, entre otras cosas, militante feminista, presidenta de la Comisión Técnica del Círculo de Inventores, asesora de empresas y periodista (ARIAS, 2017).

Por su parte, en la ciudad de La Plata (Argentina) la primera ingeniera egresada en la Universidad Nacional de La Plata y segunda ingeniera egresada en el país fue Beatriz Ghirelli con el título de "Ingeniero Mecánico Electricista" en 1938, de la cual se puede mencionar su participación en diferentes organismos: fue Directora General del IRAM (Instituto Argentino de Racionalización de Materiales), organismo público cuyos orígenes se remontan al 2 de mayo de 1935. También fue directora del IAGA (Instituto Argentino de Grasas y Aceites) y Secretaria General del CPANT (Comité Panamericano de Normas Técnicas), fundado en Brasil en 1949.

En esta línea de ideas se presenta en este trabajo, la confección, aplicación y análisis de un formulario en Google Drive, realizado a un grupo de estudiantes de ambos sexos de la FIUNLP que contestan voluntariamente y en forma anónima. El cuestionario indaga sobre la motivación por elegir carreras de ingeniería, si la motivación se despertó en la familia, en la sociedad o en la escuela, y si influyó un referente femenino en la misma; además sobre las visiones o perspectivas personales que ellos poseen para sus propios futuros profesionales. El objetivo es que los resultados obtenidos sirvan para generar y/o proponer diversas estrategias educativas, sociales y políticas que contribuyan a que más mujeres estudien carreras de ciencia y tecnología, que luego insertándose en el mundo laboral (científicos, industriales, y de poder político), sean ellas mismas un feedback y el motor para crear soluciones que achiquen la brecha de género. 


\section{MOTIVACIÓN POR ESTUDIAR CARRERAS DE INGENIERÍA: UM ESTUDIO CON PERSPECTIVA DE GÉNERO EN LA FACULTAD DE INGENIERÍA DE LA UNIVERSIDAD NACIONAL DE LA PLATA}

\section{Metodología}

La investigación se realiza en la Facultad de Ingeniería de la Universidad Nacional de La Plata (FI-UNLP) en Argentina. Esta facultad es de corte pública, posee 14 carreras de grado, y también carreras de posgrado, entre maestrías y doctorado. El número de alumnos activos es de aproximadamente 5.000 (dato a finales de 2019). La diversidad de carreras y de variedad de ellas, sumado a su gratuidad, hace que se matriculen jóvenes (varones y mujeres) de diversas ciudades de Argentina y también de Latinoamérica.

La investigación que se realiza es del tipo descriptiva y exploratoria, y resulta ser un estudio de caso (GÓMEZ Y ROQUET, 2009). Primero se presenta la situación actual relativa a la proporción según género de los estudiantes en la FI-UNLP, y también por carreras. Luego se confecciona un cuestionario en Google Drive, de carácter anónimo para ser respondido voluntariamente por estudiantes de la FI-UNLP que son convocados a responder mediante el enlace del mismo difundido por los distintos canales de conexión (redes sociales de difusión que posee la facultad, Moodle y sitios web de cátedras).

El cuestionario se eligió que sea anónimo, ya que de este modo el encuestado contestará con mejor disposición a la sinceridad. Se eligió también que sea via un Formulario de Google Drive dado la versatilidad que tiene y que es accesible por los jóvenes desde cualquier dispositivo con acceso a internet, lo cual nos aseguraría el obtener una mayor cantidad de respuestas.

El cuestionario, se estructuró del siguiente modo, pensando en que no sea excesivamente largo de contestar, que el tipo de preguntas sea apropiado, para no ofender a la persona, cuidando además el lenguaje utilizado.

La cantidad de preguntas del cuestionario es de nueve y se presentan en la Tabla 1. Algunas fueron de respuesta de texto y otras con opciones ya predeterminadas para ser más simple su análisis. La pregunta 1 indaga sobre la carrera a la que está inscripto y es de respuesta cerrada con casillas de verificación para marcar. La pregunta 2, pretende relevar su lugar de origen, siendo de respuesta cerrada con opciones para marcar. La pregunta 3, consulta si es hombre, mujer u otro. La pregunta 4, consulta sobre ¿Qué te motivó a elegir tu carrera? con respuesta de texto larga. La pregunta 5 consulta sobre ¿Cuál es la persona más importante para la elección de tu carrera? con opciones entre: "Familia”, “Amigos”, "Profesores” y “Otros”. La 
pregunta 6 , ¿Cuándo decidiste tu carrera? con opciones temporales, tiene por objetivo conocer en qué momentos los jóvenes deciden sus carreras. La pregunta 7, si ¿Existe un modelo femenino a seguir para tu carrera? pretende conocer los estereotipos de los jóvenes. La pregunta 8 ¿Cuál es tu meta en relación a tu carrera? con opciones de respuesta larga que pretende conocer que esperan de sus carreras en el ámbito profesional. La pregunta 9 ¿Dónde quieres trabajar? de respuesta cerrada, indaga sobre las preferencias de trabajo en el ámbito ingenieril.

\begin{tabular}{|c|c|c|}
\hline $\begin{array}{l}\text { Nro. de } \\
\text { pregunta }\end{array}$ & Pregunta & Tipo de respuesta - opciones \\
\hline 1 & $\begin{array}{l}\text { Carrera en la que estás } \\
\text { inscripto }\end{array}$ & $\begin{array}{l}\text { Casilla de verificación. } 14 \\
\text { opciones (las } 14 \text { carreras de la FI } \\
\text { UNLP) pueden marcar varias. }\end{array}$ \\
\hline 2 & Lugar de origen & $\begin{array}{l}\text { Varias opciones } \\
\text { - Argentina (La Plata) } \\
\text { - Argentina (otras } \\
\text { ciudades) } \\
\text { - Fuera de Argentina }\end{array}$ \\
\hline 3 & Género & $\begin{array}{l}\text { Varias opciones } \\
\text { - Hombre } \\
\text { - Mujer } \\
\text { - Otro }\end{array}$ \\
\hline 4 & $\begin{array}{l}\text { ¿Qué te motivo a elegir tu } \\
\text { carrera? }\end{array}$ & Texto de respuesta larga \\
\hline 5 & $\begin{array}{l}\text { ¿Cuál es la persona más } \\
\text { importante para la elección } \\
\text { de tu carrera? }\end{array}$ & $\begin{array}{l}\text { Varias opciones } \\
\text { - Familia } \\
\text { - Amigos } \\
\text { - Profesores } \\
\text { - Otros }\end{array}$ \\
\hline 6 & ¿Cuándo decidiste tu carrera? & $\begin{array}{l}\text { Varias opciones } \\
\text { - No recuerdo } \\
\text { - Siempre lo supe } \\
\text { - Antes de los } 12 \text { años } \\
\text { - En el secundario }\end{array}$ \\
\hline 7 & $\begin{array}{l}\text { ¿Existe un modelo femenino a } \\
\text { seguir para tu carrera? }\end{array}$ & $\begin{array}{l}\text { Varias opciones } \\
\text { - No tengo } \\
\text { - Profesoras } \\
\text { - Familia }\end{array}$ \\
\hline
\end{tabular}

Revista RBBA $\mid$ Revista Binacional Brasil Argentina 


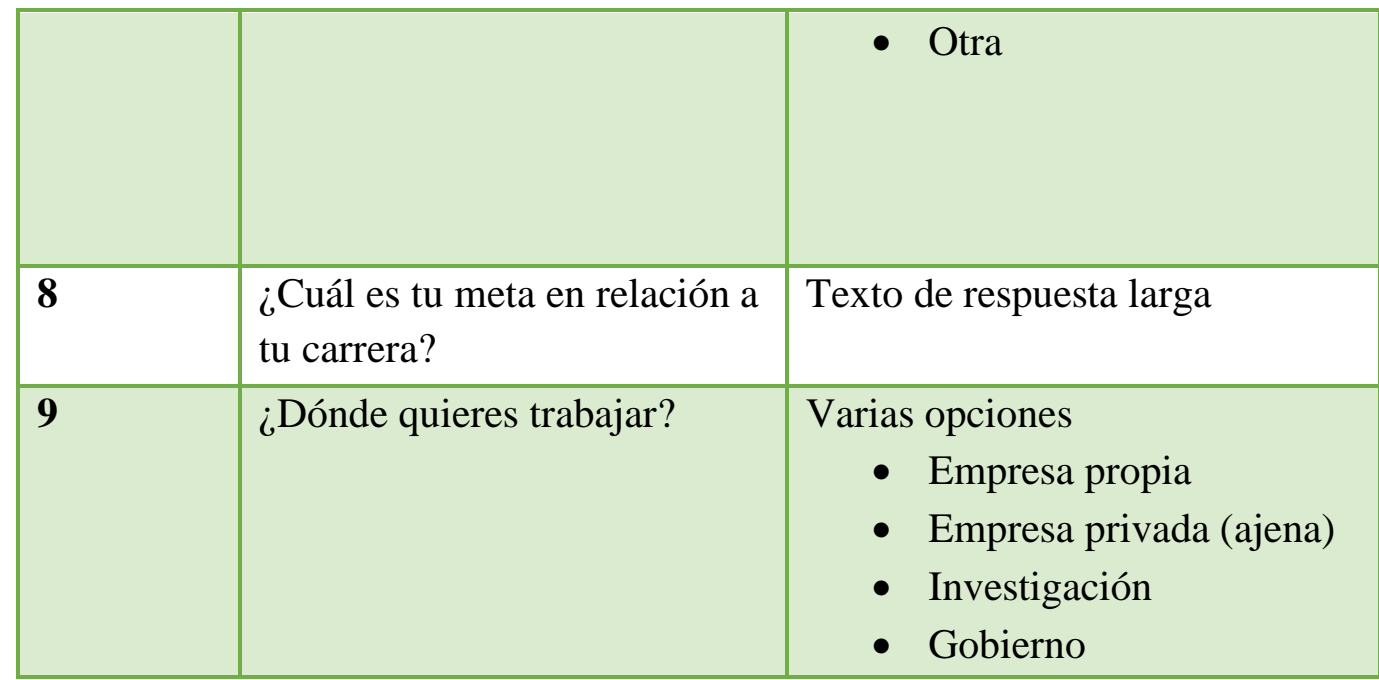

Tabla 1: Preguntas y tipo de respuestas del Cuestionario

Algunas de las preguntas del cuestionario se basan en el publicado en Ruiz y otros (2016) que fue aplicado a las alumnas inscritas a la carrera de Ingeniería Aeroespacial de la Facultad de Ingeniería de la Universidad Autónoma de Baja California, en el primer semestre del año 2014, en el que además se indagaba el modelo de desempeño futuro de las estudiantes mujeres inscritas en carreras de ingeniería, en el municipio de Mexicali. En tal trabajo los autores demuestran que no existe un modelo femenino a seguir, por lo que ellos proponen la inclusión de un modelo educativo STEM en la Facultad de Ingeniería, para incrementar la inclusión, preferencia y matrícula de mujeres en esta área del conocimiento.

\section{Antecedentes - Datos sobre género en la FI-UNLP}

En el presente trabajo se analizaron todas las carreras de ingeniería de la FI-UNLP desde el año 2015 al 2019, con excepción de la carrera de Ingeniería en Telecomunicaciones que se crea en el año 2018 e Ingeniería en Construcciones, cuyos datos se incorporan en Ingeniería Civil. A modo ilustrativo en la Figura 1 se muestran los datos en porcentaje de inscriptos totales por carrera, para el año 2017. 


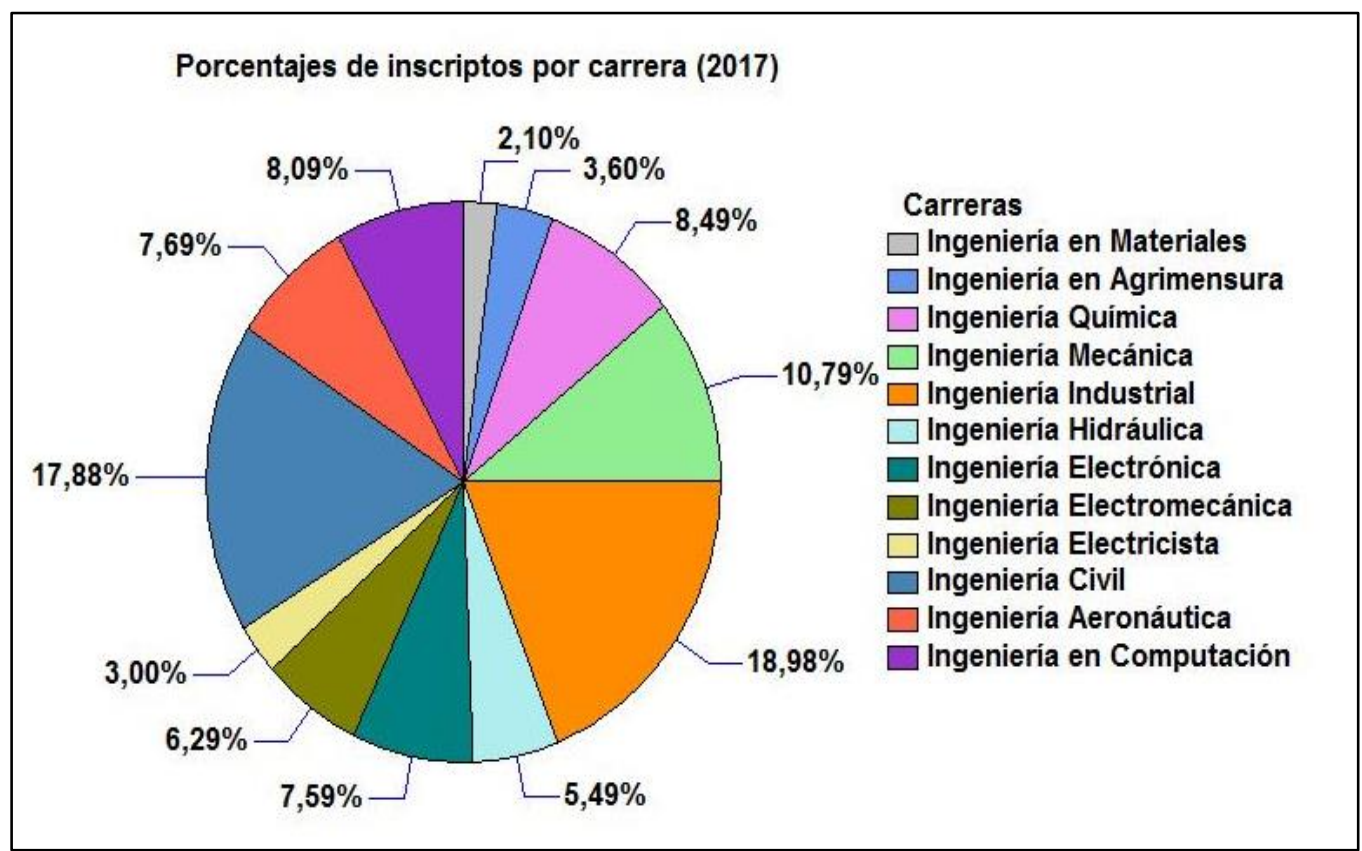

Figura 1. Porcentaje de alumnos totales inscriptos por carrera en FI-UNLP (2017).

En la FI-UNLP la proporción de mujeres es baja en relación a la cantidad de varones. En la Figura 2, se muestra una gráfica en la que se observan los datos desde el año 2015 al 2019, de la proporción de varones y de mujeres inscriptos en cada año. En promedio el $75 \%$ son varones contra un $25 \%$ de mujeres.

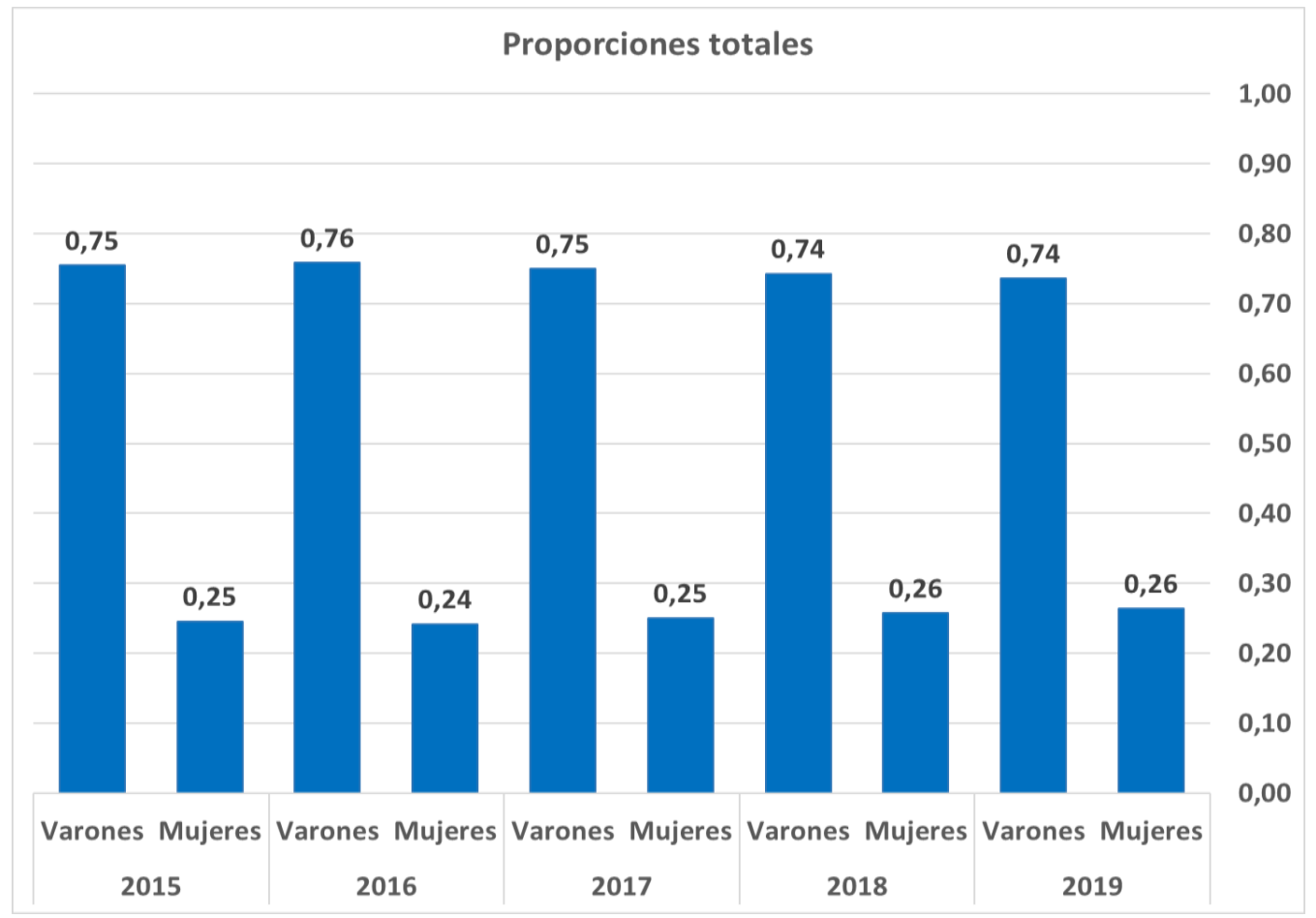

Figura 2: Proporción de mujeres y de varones inscritos en FI-UNLP del año 2015 al 2019.

Revista RBBA Revista Binacional Brasil Argentina 
En relación a las carreras que estudian las mujeres en FI UNLP, se observa en la Figura 3, según datos del año 2015 al año 2019, que Ingeniería Química es las más elegida por ellas.

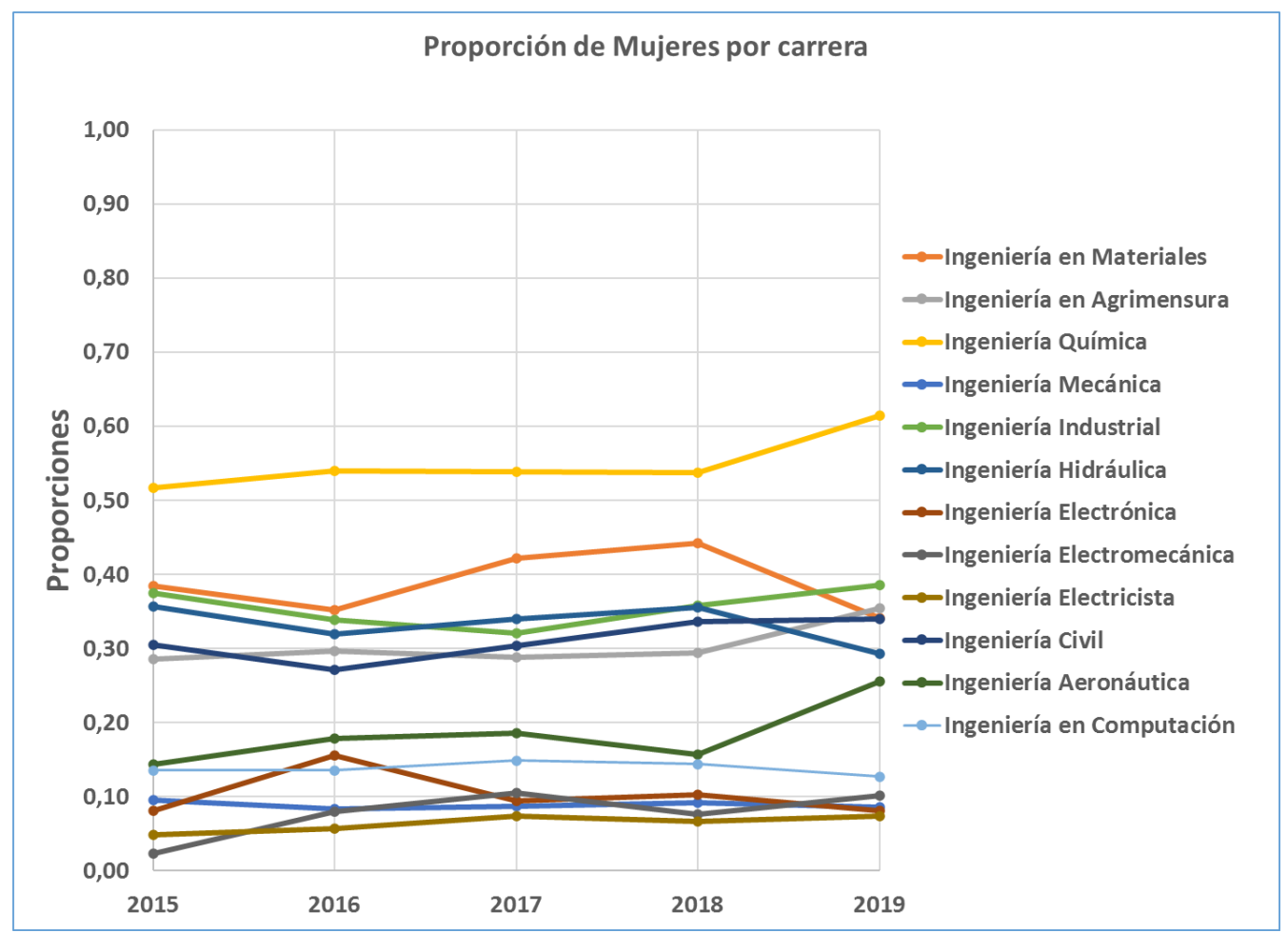

Figura 3: Proporción de mujeres por carrera del año 2015 al 2019.

Por otra parte se observa, en la misma Figura 3, que claramente aparecen dos grupos de carreras diferenciados, que le siguen en proporción de elección a la carrera de Ingeniería Química, con un porcentaje de entre el $30 \%$ al 40\%, de promedio, aparecen las carreras de Ingeniería en Materiales, Ingeniería Industrial, Ingeniería Civil, Ingeniería Hidráulica e Ingeniería en Agrimensura, respectivamente. En la tercera opción aparece sola la carrera de Ingeniería Aeronáutica con un porcentaje promedio de alrededor del $20 \%$.

Por último las carreras menos elegidas por la mujeres se conforman por el grupo de Ingeniería en Computación, Ingeniería Electrónica, Ingeniería Mecánica, Ingeniería Electromecánica e Ingeniería Electricista, constituyendo entre un 5\% al 10\% en la elección de las mujeres.

De lo anterior se puede desprender que la carrera más elegida, Ingeniería Química, está íntimamente ligada al ámbito de la investigación y desarrollo, luego la siguiente orientación en 


\section{MOTIVACIÓN POR ESTUDIAR CARRERAS DE INGENIERÍA: UM ESTUDIO CON PERSPECTIVA DE GÉNERO EN LA FACULTAD DE INGENIERÍA DE LA UNIVERSIDAD NACIONAL DE LA PLATA}

la elección de las carreras de ingeniería por parte de las mujeres esta dado hacia el campo de los proyectos, diseños, y trabajo de administración en las empresas, siendo menos optadas las carreras que están más asociada al trabajo técnico mecánico y eléctrico, que se puede vincular al mantenimiento, trabajo en planta, etc.

\section{Resultados del Cuestionario}

La cantidad de respuestas obtenidas del cuestionario fue de 204. A continuación se detallan en la Tabla 2, las preguntas y las respuestas. Para estas se consideraron las respuestas totales (varones y mujeres: 204), y luego sólo las respuestas de las mujeres que son un total de 84. Cabe aclarar que en las respuestas aparece la carrera de Ingeniería Aeroespacial, esto es porque en el año 2020 la carrera de Ingeniería Aeronáutica cambia su nombre a Aeroespacial, siendo que la presente encuesta fue realizada al comienzo del año 2020.

Las respuestas, a las preguntas que eran cerradas con opciones, se muestran los porcentajes obtenidos, y de las preguntas de respuesta larga, se utilizó un analizador de palabras para obtener las más frecuentes. Además, en la Tabla se han resaltado los mayores valores para cada categoría.

\begin{tabular}{|l|l|l|c|c|}
\hline $\mathbf{N}^{\circ}$ & Pregunta & Respuestas: & Todos & $\begin{array}{c}\text { Sólo } \\
\text { mujeres }\end{array}$ \\
\hline $\mathbf{1}$ & Carrera en la que estás & Civil: & $21 \%$ & $8 \%$ \\
& inscripto & Aeronáutica: & $2 \%$ & $2 \%$ \\
& & Hidráulica: & $5 \%$ & $8 \%$ \\
& & Computación: & $9 \%$ & $4 \%$ \\
& & Materiales: & $5 \%$ & $0 \%$ \\
& & Agrimensura: & $3 \%$ & $3 \%$ \\
& & Aeroespacial: & $1 \%$ & $1 \%$ \\
& & Electrónica: & $20 \%$ & $4 \%$ \\
& & Telecomunicaciones: & $3 \%$ & $1 \%$ \\
& & Electricista: & $2 \%$ & $0 \%$ \\
& & Mecánica: & $25 \%$ & $41 \%$ \\
& & Química: & $8 \%$ & $10 \%$ \\
& & Industrial: & $1 \%$ & $4 \%$ \\
\hline \multirow{2}{*}{} & Lugar de origen & Electromecánica: & $50 \%$ & $51 \%$ \\
& & Argentina (La Plata): & $42 \%$ & $39 \%$ \\
& & Argentina (otras ciudades): & $8 \%$ & $10 \%$ \\
\hline
\end{tabular}

\begin{tabular}{l|l} 
Revista RBBA & Revista Binacional Brasil Argentina
\end{tabular} 


\begin{tabular}{|c|c|c|c|c|}
\hline 3 & Género & $\begin{array}{l}\text { Hombre: } \\
\text { Mujer: } \\
\text { Otro: }\end{array}$ & \multicolumn{2}{|c|}{$\begin{array}{c}59 \% \\
41 \% \\
0 \%\end{array}$} \\
\hline 4 & $\begin{array}{l}\text { ¿Qué te motivo a elegir } \\
\text { tu carrera? }\end{array}$ & Para las mujeres solamente: & \multicolumn{2}{|c|}{$\begin{array}{l}\text { "salida laboral" } \\
\text { "me gusta" "me parece } \\
\text { interesante" } \\
\text { "poder crear", } \\
\text { "construir" } \\
\text { "interés por las ciencias" } \\
\text { "salida laboral" } \\
\text { "me parece interesante" } \\
\text { "el dinero" } \\
\text { "amplio y versátil campo } \\
\text { laboral". }\end{array}$} \\
\hline 5 & $\begin{array}{l}\text { ¿Cuál es la persona } \\
\text { más importante para la } \\
\text { elección de tu carrera? }\end{array}$ & $\begin{array}{l}\text { Familia: } \\
\text { Amigos: } \\
\text { Profesores: } \\
\text { Otros: }\end{array}$ & $\begin{array}{c}52 \% \\
5 \% \\
17 \% \\
26 \%\end{array}$ & $\begin{array}{r}42 \% \\
4 \% \\
20 \% \\
34 \%\end{array}$ \\
\hline 6 & $\begin{array}{l}\text { ¿Cuándo decidiste tu } \\
\text { carrera? }\end{array}$ & $\begin{array}{l}\text { No recuerdo: } \\
\text { Siempre lo supe: } \\
\text { Antes de los } 12 \text { años: } \\
\text { En el secundario: }\end{array}$ & $\begin{array}{c}8 \% \\
7 \% \\
5 \% \\
80 \%\end{array}$ & $\begin{array}{l}3 \% \\
7 \% \\
3 \% \\
87 \%\end{array}$ \\
\hline 7 & $\begin{array}{l}\text { ¿Existe un modelo } \\
\text { femenino a seguir para } \\
\text { tu carrera? }\end{array}$ & $\begin{array}{l}\text { No tengo: } \\
\text { Profesoras: } \\
\text { Familia: } \\
\text { Otra: }\end{array}$ & $\begin{array}{l}63 \% \\
19 \% \\
11 \% \\
7 \%\end{array}$ & $\begin{array}{l}55 \% \\
21 \% \\
16 \% \\
7 \%\end{array}$ \\
\hline 8 & $\begin{array}{l}\text { ¿Cuál es tu meta en } \\
\text { relación a tu carrera? }\end{array}$ & Palabras frecuentes: & \multicolumn{2}{|c|}{$\begin{array}{l}\text { "recibirme" } \\
\text { "poder trabajar" } \\
\text { "aprender" } \\
\text { "ayudar a la sociedad" } \\
\text { "trabajar en la industria" } \\
\text { "ayudar a mis padres" } \\
\text { "superarme" } \\
\text { "mejorar la calidad de } \\
\text { vida" } \\
\text { "perfeccionarme en el } \\
\text { exterior". }\end{array}$} \\
\hline 9 & $\begin{array}{l}\text { ¿Dónde quieres } \\
\text { trabajar? }\end{array}$ & $\begin{array}{l}\text { Empresa propia } \\
\text { Empresa privada (ajena): } \\
\text { Investigación } \\
\text { Gobierno: }\end{array}$ & $\begin{array}{c}33 \% \\
43 \% \\
18 \% \\
6 \%\end{array}$ & $\begin{array}{r}30 \% \\
52 \% \\
12 \% \\
6 \%\end{array}$ \\
\hline
\end{tabular}

Tabla 2: Respuestas del cuestionario. 


\section{MOTIVACIÓN POR ESTUDIAR CARRERAS DE INGENIERÍA: UM ESTUDIO CON PERSPECTIVA DE GÉNERO EN LA FACULTAD DE INGENIERÍA DE LA UNIVERSIDAD NACIONAL DE LA PLATA}

\section{Análisis}

El cuestionario fue contestado con 204 estudiantes, con un 59\% de varones y un $41 \%$ de mujeres, de los cuales se encontró que el mayor porcentaje que lo respondieron fue por alumnas de Ingeniería Química, que dentro del conjunto de las mujeres representaron un 41\%, siendo además en gran mayoría provenientes de la ciudad de La Plata, seguido de otras ciudades de Argentina, y en menos proporción de fuera del país.

En relación a la pregunta ¿Qué te motivo a elegir tu carrera? Las respuestas más frecuentes se relacionan con mencionar: "salida laboral", "me gusta", "poder crear", “construir", "interés por las ciencias", "me parece interesante”, "el dinero", "amplio y versátil campo laboral".

Para la pregunta ¿Cuál es la persona más importante para la elección de tu carrera? Se encontró que la familia (52\% - 41\%) es el entorno social que más influye en la elección de la carrera seguido de los profesores $(16 \%$ - 20\%). Este resultado es similar al encontrado en el trabajo referenciado realizado en México, en relación a la pregunta ¿Cuál es la persona más importante para la elección de tu carrera? en el que encuentran que el $49 \%$ tienen apoyo por parte de sus padres para estudiar, el 39\% expresó sentirse apoyado por sus amigos y el resto por sus maestros, con el porcentaje más bajo.

Sobre cuándo deciden estudiar ingeniería, el $80 \%$ menciona que ocurre durante el colegio secundario.

En relación a la pregunta relacionada a si existe un modelo femenino a seguir, el $63 \%$ (total) y el $55 \%$ de las mujeres menciona que no posee. En comparación al trabajo realizado en México, los autores encuentran que el $75 \%$ de las encuestadas dijeron no tener alguno, el 19\% expresó sentirse motivado por sus maestras, y sólo el $6 \%$ afirmó tener una familiar ingeniera. Encontrándose resultados similares, que indicarían un patrón en relación a que los jóvenes no poseen un modelo a seguir que los haya motivado en la elección de sus carreras.

En relación a la pregunta ¿Cuál es tu meta en relación a tu carrera?, las frases más frecuentes tienen que ver con la superación personal y académica, ayudar en sus familias, a la sociedad y mejorar su calidad de vida.

En relación a la pregunta 9, en el trabajo de México sobre ¿Dónde quieres trabajar? se obtuvo que el $50 \%$ expresó desear trabajar en una empresa, 32\% manifestó tener su propia empresa, $6 \%$ expresó su interés por desarrollarse en la investigación, $6 \%$ demostró un interés 


\section{MOTIVACIÓN POR ESTUDIAR CARRERAS DE INGENIERÍA: UM ESTUDIO CON PERSPECTIVA DE GÉNERO EN LA FACULTAD DE INGENIERÍA DE LA UNIVERSIDAD NACIONAL DE LA PLATA}

por la docencia y otro $6 \%$ por trabajar como empleado de alguna instancia gubernamental. En el cuestionario de este trabajo se obtuvo que el $76 \%$ aproximadamente quisiera trabajar en una empresa (pública o privada) y solo el resto entre investigación y gobierno.

\section{Conclusión}

En este trabajo se presenta un breve panorama a nivel mundial, latinoamericano y local sobre la problemática relacionada a la históricamente baja cantidad de mujeres que estudian carreras de ciencias e ingeniería. Si bien este es un problema complejo, en esta investigación intentamos colaborar con comprenderlo y realizar aportes a partir de los resultados realizados a un grupo de estudiantes de la FI-UNLP.

Para afrontar esta problemática que está vinculada con las brechas de género, los estereotipos que existen acerca de que se piensa a las carrearas de ingeniería son para hombres, entre otras, se requieren acciones de diferentes actores, políticos, educativos y sociales.

En este contexto, es importante que las instituciones de educación superior, que tienen cierta influencia, se centren en generar acciones para lograr que una mayor cantidad de mujeres logren acceder a estudiar carreras de ciencias e ingeniería, presentando proyectos e iniciativas que sirvan de puente entre la política pública y las acciones institucionales y la sociedad.

Los resultados del cuestionario realizado a 204 estudiantes de la FI-UNLP, muestran que:

- Las mujeres deciden estudiar carreras de ingeniería durante la etapa en la que están en el colegio secundario. Esto es un concepto clave, dado que las políticas/estrategias deberían tener como objetivo de ser implementadas en esas etapas ya que allí es donde las mujeres se motivarían por estudiar carreras de ingeniería.

- La falta de modelos o referentes femeninos a seguir en ciencias e ingeniería, tanto para los varones como para las mujeres.

- La familia es el mayor referente y apoyo de los jóvenes para elegir sus carreras.

En este sentido podemos pensar en las siguientes acciones: 


\section{MOTIVACIÓN POR ESTUDIAR CARRERAS DE INGENIERÍA: UM ESTUDIO CON PERSPECTIVA DE GÉNERO EN LA FACULTAD DE INGENIERÍA DE LA UNIVERSIDAD NACIONAL DE LA PLATA}

- Generar políticas de igualdad de género en los contextos educativos que sirvan para establecer estrategias a largo plazo para terminar con la brecha de género.

- Promover la igualdad de género desde las prácticas docentes en los distintos niveles educativos primarios y secundarios, en todas las asignaturas, tanto de ciencias como otras. Incluir actividades en el enfoque STEM en las instituciones educativas.

- Apoyar la creación de asociaciones de mujeres en torno a las áreas disciplinares de ciencia e ingeniería que faciliten el diálogo entre grupos académicos y profesionales.

- Incluir modelos femeninos en los niveles educativos. En particular en la UNLP se recomienda tomar de referente a Beatriz Ghirelli y su importante trabajo, y proponerla como figura de referencia para futuras generaciones, incluso promover que la facultad difunda su trayectoria académica y personal en las charlas que se hacen para las escuelas del nivel secundario.

- Proponer diversas acciones para que las mujeres se inclinen por otras carreras de ingeniería además de las más populares entre ellas como son Ingenieria Química e Industrial.

- Motivar el interés por la ciencia y la ingeniería, en las jóvenes en sus entornos familiares y de amistades, proponiéndoles por ejemplo diversas actividades lúdicas de esparcimiento, de lectura, visita de museos, museos de ciencias, museos interactivos, vista de documentales y películas, entre otros, de modo de lograr despertar el interés de ellos en la temática.

\section{REFERENCIAS}

ARANGO GAVIRIA, L. G. Género e ingeniería: la identidad profesional en discusión. Revista Colombiana de Antropología, Bogotá: Instituto Colombiano de Antropología e Historia, Colombia, v. 42, 2006, p.129-156.

ARIAS, A. C. Mujeres universitarias en la Argentina. Algunas cuestiones acerca de la Universidad Nacional de La Plata en las primeras décadas de siglo XX». Trabajo Final de la Especialización en Educación en Géneros y Sexualidades Facultad de Humanidades y Ciencias de la Educación Universidad Nacional de La Plata, 2017.

BONDER, G. Las nuevas tecnologías de información y las mujeres: reflexiones necesarias. CEPAL, 2002. 


\section{MOTIVACIÓN POR ESTUDIAR CARRERAS DE INGENIERÍA: UM ESTUDIO CON PERSPECTIVA DE GÉNERO EN LA FACULTAD DE INGENIERÍA DE LA UNIVERSIDAD NACIONAL DE LA PLATA}

CONFEDI. "Matilda y las mujeres en ingeniería en América Latina". Universidad FASTA Ediciones, Argentina, 2018.

GARCÍA, Y.; GONZÁLEZ, D. S. M. R.; OVIEDO, F. B. Actividades STEM en la formación inicial de profesores: nuevos enfoques didácticos para los desafíos del siglo XXI. Diálogos educativos, 2017, no 33, p. 35-46.

GARCÍA-HOLGADO, A., CAMACHO DÍAZ, A., \& GARCÍA-PEÑALVO, F. J. La brecha de género en el sector STEM en América Latina: Una propuesta europea, 2019.

GÓMEZ, D. R.; ROQUET, J. V. Metodología de la investigación. Universitat Oberta de Catalunya, 2009.

LALANNE, T., \& SOLANET, M. (2019). ¿Dónde están las mujeres en las carreras de ingeniería?: el caso argentino.

ROSSITER, M. W. The Matthew Matilda effect in science. Social studies of science, 1993, vol. 23, no 2, p. 325-341.

RUIZ, M. A. O., CÓRDOBA, E. C., SALAS, B. V., \& WIENNER, M. S. La motivación de las mujeres por las carreras de ingeniería y tecnología. Entreciencias: Diálogos en la sociedad del conocimiento, 4(9), 89-96, 2016.

ONU Mujeres. (2019). Generación Igualdad: Por los derechos de las mujeres y un futuro igualitario. 2019. https://www.unwomen.org/es/digitallibrary/publications/2019/05/ generation-equality Recuperado el 20 de mayo de 2019.

UNESCO, 2019. Descifrar el código: la educación de las niñas y las mujeres en ciencias, tecnología, ingeniería y matemáticas (STEM). Paris, Francia 2019. Título original: Cracking the code: Girls' and women's education in science, technology, engineering and mathematics (STEM) Publicado en 2017 por la Organización de las Naciones Unidas para la Educación, la Ciencia y la Cultura. 2019. Recuperado el 20 de mayo de https://unesdoc.unesco.org/ark:/48223/pf0000366649.locale=es Recuperado el 20 de mayo de 2019. 


\section{ACERCA DE LAS AUTORAS}

Viviana Angélica Costa es Doctora en Enseñanza de las Ciencias, Facultad de Ciencias Exactas, Universidad Nacional del Centro de la Provincia de Buenos Aires. Magister en Simulación Numérica y Control, Facultad de Ingeniería, Universidad de Buenos Aires. Licenciada en Matemática, Facultad de Ciencias Exactas, Universidad Nacional de La Plata. Coordinadora de la UIDET Investigación en Metodologías Alternativas para la Enseñanza de las Ciencias, Facultad de Ingeniería, Universidad Nacional de la Plata (UIDET IMApEC). Profesora Adjunta Dedicación Exclusiva, Departamento de Ciencias Básicas, Facultad de Ingeniería, Universidad Nacional de la Plata. Profesora Adjunta Dedicación Simple, Departamento de Turismo, Facultad de Ciencias Económicas, Universidad Nacional de la Plata. Integrante del Nucleo De Investigación en Educación en Ciencia y Tecnología (NIECyT), Universidad Nacional del Centro, Argentina.

Dirección Electrónica: vacosta @ing.unlp.edu.ar ORCID Id 0000-0003-1782-5378

María Valeria Calandra. Licenciada en Matemática, Facultad de Ciencias Exactas, Universidad Nacional de La Plata. Profesora Adjunta Ordinaria con Dedicación Exclusiva - Probabilidades y Estadística, Departamento de Ciencias Básica, Facultad de Ingeniería, Universidad Nacional de La Plata. Profesora Adjunta Ordinaria Dedicación Simple, Departamento de Matemática, Facultad de Ciencias Exactas, Universidad Nacional de La Plata. UIDET Grupo de Aplicaciones Matemáticas y Estadísticas de la Facultad de Ingeniería (UIDET Gamefi), Departamento de Ciencias Básicas, Facultad de Ingeniería, Universidad Nacional de La Plata.

Dirección Electrónica: mava@mate.unlp.edu.ar ORCID Id 0000-0002-5477-4832

Juana Inés Gallego Sagastume. Ingeniera en Electrónica, Facultad de Ingeniería, Universidad Nacional de La Plata. Alumna de la Maestría en Física Contemporánea, Facultad de Ciencias Exactas, Universidad Nacional de La Plata. Profesora y Jefa de Trabajos Prácticos en las Cátedras de Física, Departamento de Ciencias Básicas, Facultad de Ingeniería, Universidad Nacional de la Plata. Escritora y organizadora del Encuentro de Ciencia Ficción Pórtico. 
Capacitadora en el movimiento guía-scout (WAGGGS), especialmente en el área de Aire Libre.

Dirección Electrónica: chinchiya@gmail.com

ORCID Id 0000-0003-0487-404X

Recebido em: 25/03/2020

Aprovado em: 04/05/2020

Publicado em: 01/07/2020 